\title{
A NEW QUALITY FUNCTION DEPLOYMENT FUZZY EVALUATION MATRIX FOR SOFTWARE DESIGN PLANNING BY USING ANALYTIC HIERARCHY PROCESS AND FUZZY SET THEORY
}

\author{
Kuo-Sui Lin and William McEwan \\ Quality Centre, \\ University of Paisley, Paisley, Scotland, UK. \\ E-Mail Lin_qc0@Paisley.ac.uk \\ Kwo-Jean Farn \\ Computer \& Communication Research Laboratories, \\ Industrial Technology Research Institute, Hsinchu, Taiwan, R.O.C. \\ 720460@ITRIOAI.ITRI.ORG.TW
}

\begin{abstract}
Customer satisfaction is the purpose of Total Quality Management (TQM). In order to early evaluate and assure the quality of software products for customer satisfaction, we have illustrated the application of Quality Function Deployment (QFD) to solicit and deploy customer requirements into measurable software design attributes. We further have proposed a new QFD fuzzy evaluation matrix by using Analytic Hierarchy Process (AHP) and fuzzy set theory to prioritise the software design attributes and identify the attributes that are the most important and fundamental to customer satisfaction. An example is illustrated and demonstrated that the new QFD fuzzy evaluation matrix is realistic for the prioritisation of software design attributes.
\end{abstract}

\section{Introduction}

Becoming more aware of the importance of earlier stages in software engineering life cycle, the concurrent design concept should be emphasised to early evaluate and assure the quality of software products, focusing on customer satisfaction and achieving the purpose of Total Quality Management (TQM). In this regard, the authors will use the Quality Function Deployment (QFD) evaluation matrix as a quality assurance (QA) tool to translate customer requirements, track software design attributes and identify design attributes that are critical to customer satisfaction.

The quality of software products can be modelled by software quality models. Many researchers have proposed different software quality models and defined different software quality attributes to represent and evaluate the quality of software products $[1,2,3]$. In this paper, the software quality attributes proposed by Deutsch and Willis [3] are used for the evaluation guidelines of software design plan. These attributes will be shown in the illustrated examples.

Through the application of the QFD evaluation matrix, the quality design attributes which present the extra attraction for customer satisfaction are identified. The process of evaluating quality of software products is a multiple attribute decision making process involving a set of customer requirements for which the information can be imprecise and subjective. Therefore, we propose a new QFD fuzzy evaluation matrix by using AHP and fuzzy set theory to evaluate imprecise and subjective interrelationships between customer requirements and design attributes more realistically. The fuzzy ratings in the new evaluation matrix accept all the membership values of all appraisal levels.

\section{A QFD Evaluation Matrix for Software Design Planning}


QFD in software development starts with a comprehensive understanding of customer requirements and then arranges it so that it could be used for planning the software design direction by deploying design attributes, and prioritising design attributes. Once the design plan has been consented and approved, one begins the actual design work.

\subsection{Quality Function Deployment}

QFD was first systematised in Japan by Y. Akao in mid-1970 at Mitsubishi's Kobe shipyards. It is a multiphase product (service) development process for deploying customer requirements based on interfunctional cooperation and communication among members from marketing, $R \& D$, engineering, and manufacturing.

QFD is an increasingly popular structured method for converting the customers' demands into "quality characteristics" and developing a design quality for the finished product by systematically deploying the relationships between the demands and the characteristics, starting with the quality of each functional

component and expending the deployment to the quality of each part and process [4].

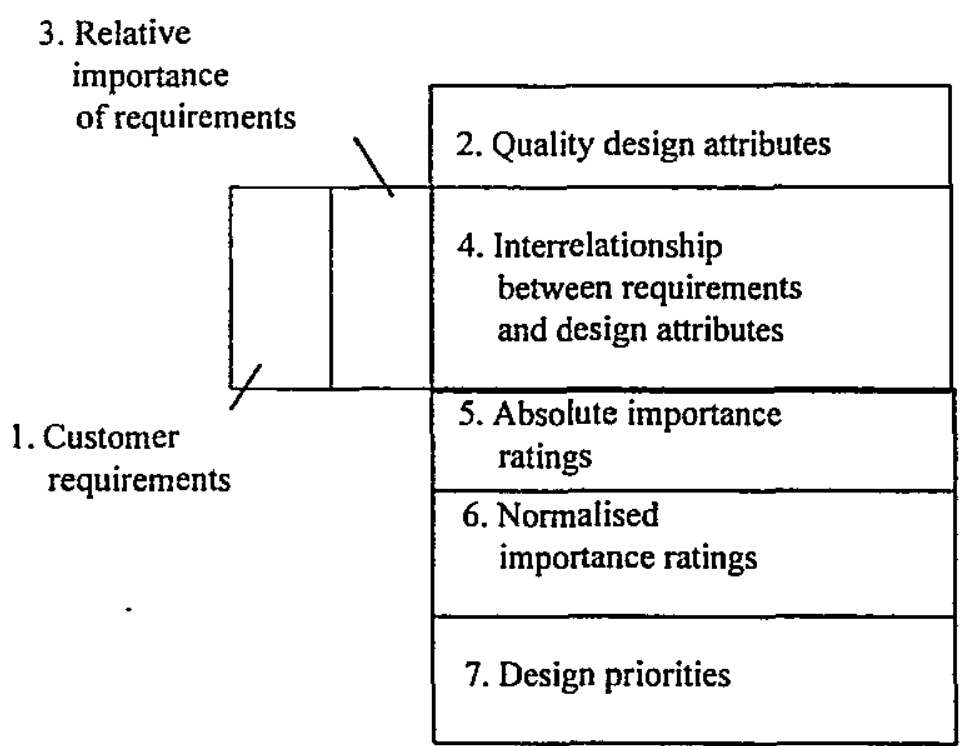

Fig. 1. The QFD House of Quality

A lot of literature is proposed by different authors with different versions describing how to make QFD concrete and workable $[5,6,7]$. In spite of the differences, the QFD iteration process propagating the "whats" into "hows" and into "how muchs" by an input-output mapping is in common. This iteration process tries to convert the vague requirements into proper measurable design attributes. Essentially, the house of quality in this paper (see Figure 1) is used in the following steps:

Step 1 Identify and structure a set of customer requirements, the "whats".

Step 2 Deploy a set of quality design attributes, the "hows", from the customer requirements.

Step 3 Assign a set of importance weightings to the set of customer requirements, if different requirements carry different relative importance.

Step 4 Map the degree of interrelationship, relationship ratings, for the "whats vs. hows", between the customer requirements and the quality design attributes.

Step 5 Aggregate the importance weightings and the relationship ratings into absolute importance ratings, "how muchs".

Step 6 Transfer the absolute importance ratings into normalised importance ratings.

Step 7 Prioritise the quality design attributes and identify the critical design attributes with most leverage for satisfying customer requirements.

\subsection{QFD Evaluation and Prioritisation Approach}


Following the steps of the house of quality introduced in the last section, we will explain the QFD evaluation and prioritisation approach in this section.

A set of customer requirements $C$ is identified in step 1 and a set of design attributes $A$ is deployed in step 2, where

$$
\begin{aligned}
& C=\left\{C_{l}, i=1, \ldots, m\right\} \\
& A=\left\{A_{j}, j=1, \ldots, n\right\} .
\end{aligned}
$$

Assume that each customer requirement $C_{i}$ in $C$ has a different importance weighting $W_{l}$ in $W$. The values of importance weighting set $W$ can be described by $W=\left[w_{1}, w_{2}, \ldots, w_{m}\right]$, where $i=1 \ldots m, w_{i} \in[0,1]$ and

$\sum_{i=1}^{m} w_{i}=1$. Different values of weightings reflect various degrees of importance of customer requirements. The values of weightings can be derived by pairwise comparison of the elements in $C$ to determine the reciprocal matrix and the eigenvector. This is based on Saaty's Analytic Hierarchy Process (AHP) [8].

In order to map the degree of interrelationship, we use the relationship rating $r_{i j}$ between the customer requirement $C_{i}$ and the to design attribute $A_{j}, i=12 \ldots m, j=1 \ldots n$. The ratings are represented by comparable crisp scale, thus, the characteristics of design attribute $A_{j}$ with respect to all customer requirements can be represented as:

$$
A_{j}=\left\{\left(C_{1}, r_{1 j}\right),\left(C_{2}, r_{2 j}\right), \ldots,\left(C_{m}, r_{m j}\right)\right\} .
$$

By using the matrix notation, the rating set of $A_{j}$ can be represented by the following matrix:

$$
R_{j}=\left[r_{1 j}, r_{2 j}, \ldots, r_{m j}\right] \text {. }
$$

In step 5 , the absolute importance ratings $S_{j}, j=1 \ldots n$, can be derived by aggregating the weightings and the relationship ratings according to the following formula:

$$
\begin{aligned}
S_{j} & =W \cdot R_{j}^{\top} \\
& =\left[w_{1}, w_{2}, \ldots, w_{m}\right] \cdot\left|\begin{array}{c}
r_{1 j} \\
r_{2 j} \\
\cdot \\
\cdot \\
r_{m j}
\end{array}\right|,
\end{aligned}
$$

where $S_{j}=\sum_{i=1}^{m} w_{i} \cdot r_{i j}$, for $j=1 \ldots n$.

Hence, the normalised importance ratings $S_{j}, j=1 \ldots n$, can be derived according to the following formula:

$$
S_{j}^{\prime}=S_{j} / \sum_{j=1}^{n}\left|S_{j}\right|
$$

\subsection{Illustrated Example}

The application of QFD evaluation matrix in a software design plan is explained in this example and shown in Figure 2. Firstly, seven major customer requirements are solicited. The solicited customer requirements reflect the voice of the software quality needs. Then software quality factors defined by Deutsch and Willis [3] are selected as software design attributes to map the customer requirements to these attributes. In the importance weightings assignment step, a set of importance's weightings, $W$, expressing the degrees of importances of customer requirements, is derived by using the AHP method. 


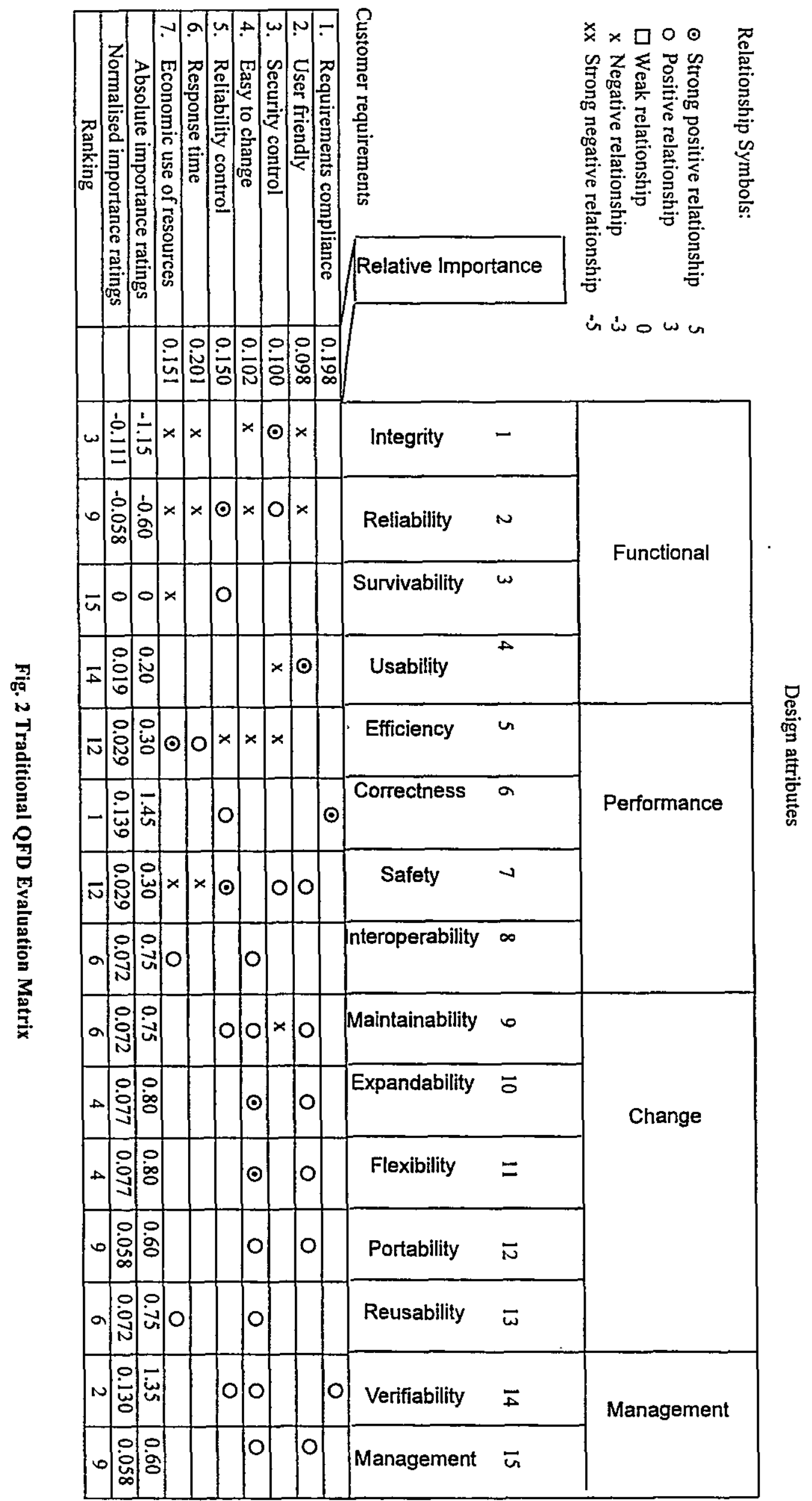


$w_{i} \in W$ is the importance weighting of requirement $C_{i}$. Figure 3 shows the evaluation hierarchy of the AHP method.

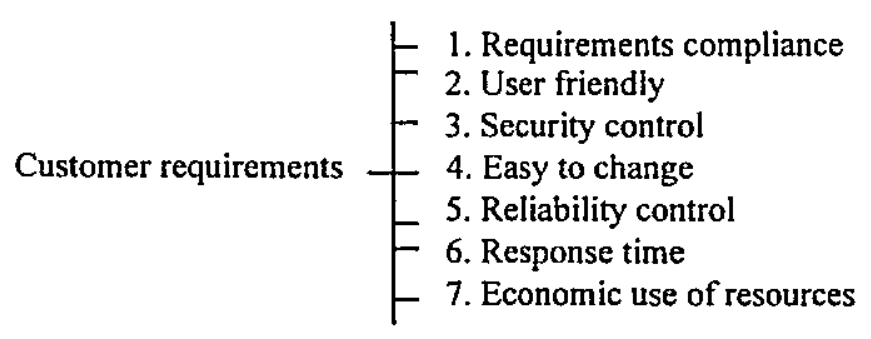

Fig. 3 Evaluation Hierarchy of the AHP Method

In the relationship rating step, the impact of the fulfilment of quality design attributes on the satisfaction of customer requirements is quantified through the use of $5,3,0,-3$, or -5 scale to denote "strong positive relationship", "positive relationship", "weak relationship", "negative relationship" or "strong negative relationship", and symbolised by " $O$ ", " $O$ ", " $\square$ ", " $x$ " or " $x x$ " respectively. Therefore, according to Eq.(1), the absolute importance rating of $A_{1}$ can be derived as follows:

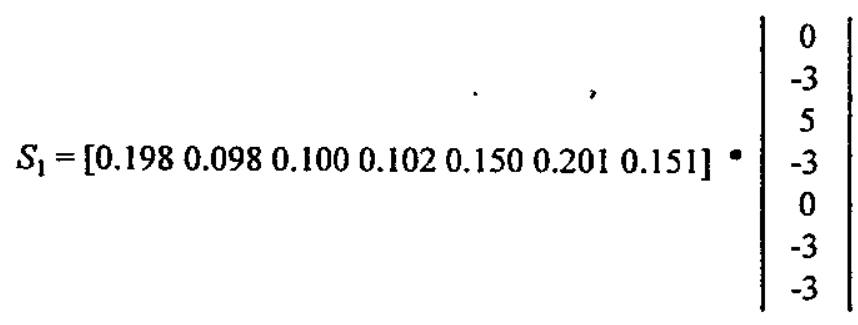

$$
\begin{aligned}
& =-1.15 \text {. }
\end{aligned}
$$

In a similar manner, we can obtain the absolute importance ratings of other attributes as illustrated in Figure 2. According to Eq (2), the normalised importance rating of $A_{1}$ can be obtained as

$$
S_{1}{ }^{\prime}=-1.15 / 10.4=-0.111 \text {. }
$$

In a similar manner, the normalised importance ratings of other attributes can be derived as illustrated in Figure 2. Therefore, the order of ranking of the design attributes is derived as illustrated in Figure 2. The positive(negative) scores mean that the customer satisfaction will be affected positively(negatively) and deserve focus design efforts.

\section{A New QFD Fuzzy Evaluation Matrix for Software Design Planning}

In the last section, the degrees of interrelationship $f_{i j}$ between customer requirements $C_{i}, i=1 \ldots m$, and design attributes $A_{j}, j=1 \ldots n$, are represented by a relationship rating set. The elements of the relationship rating set are valued by crisp values. For example, those elements that belong to one specific appraisal level, e.g., positive relationship, are valued by the specific crisp value of 3 . The abrupt and unambiguous crisp values are not plausible to exhibit the imprecise and subjective relationship ratings between customer requirements and design attributes. In this section, therefore, we will use fuzzy set theory to introduce fuzziness by eliminating the abrupt and unambiguous boundaries dividing members from nonmembers of one specific appraisal level. The membership value in the fuzzy set corresponds to the degree to which each appraisal level approximates the imprecise and subjective concept of "strong positive relationship", "positive relationship", "weak relationship", "negative relationship", or "strong negative relationship".

\subsection{Fuzzy Muitiple Attribute Decision Making and It's Underlying Concepts}


A fuzzy multiple attribute decision making method comprises a two-phase process. The first phase is to find fuzzy utilities of alternatives. The second phase is to apply a fuzzy ranking method to determine the ranking order of alternatives.

Let $A=\left\{A_{j}, j=1, \ldots, n\right\}$ be a finite set decision alternatives and $C=\left\{C_{i}, i=1, \ldots, m\right\}$ be a finite set of criteria (attributes) with which alternative performances are measured. $R=\left(r_{i j}\right)_{m \times n}$ is the matrix of fuzzy performance ratings of alternative $A_{j}$ with respect to criterion $C_{i}, i=1, \ldots, m, j=1, \ldots, n . W=\left(w_{b}, \ldots, w_{m}\right)$ is a vector of fuzzy relative importance weightings of criteria. A fuzzy multiple attribute decision making method is used to find an optimal alternative with highest utility to the decision maker. The utility function aggregates the fuzzy performance ratings with fuzzy importance weightings representing how well alternatives satisfy the decision maker's utility.

However, in the real environment, the alternative performance ratings, the relative importance weightings and the resulted utilities are no longer crisp numbers; they are fuzzy quantities. Fuzzy quantities do not provide comparable scores for the decision maker's evaluation as crisp numbers do.

In this paper, the fuzzy utilities are obtained by composition method defined in definition 3 . The fuzzy utilities are fuzzy quantities instead of numerical quantities. Therefore, the fuzzy transformation method defined in definition 4 is used to transform the fuzzy quantities into numerical scores. The ranking order of alternatives can be obtained by comparing the resulted numerical scores of alternatives. In what follows, we shall introduce some underlying concepts of fuzzy multiple attribute decision making.

\section{Definition 1. Classical Sets}

Let $X$ be a set of objects, called the universe of discourse, whose generic elements are denoted by $x, x \in X$. A classical set (crisp set) $\mathrm{H}$ in $X$ is characterised by a membership function $\mu_{H}: X \rightarrow\{0,1\}, \mu_{H}(x) \in\{0,1\}$ is the membership value of $x$ in $\mathrm{H}$,

$$
\mu_{H}(x)=\left\{\begin{array}{l}
1, \text { iff } x \in H, \\
0, \text { iff } x \notin H .
\end{array}\right.
$$

The objects satisfy precise properties required for membership values 0 or 1 .

Because $\mu_{H}$ map all real number $x \in X$ onto the two points $\{0,1\}$, a classical set corresponds to a 2-valued logic; An object is, either in a set or not in a set and cannot partially belongs to a set is the limitation of a classical set. To overcome the limitation, a fuzzy set that admits partial membership was introduced by Zadeh (8) in 1965 to deal with the fuzziness which is difficult to deal with by a classical set.

\section{Definition 2. Fuzzy Sets}

A fuzzy set $F$ in the universe of discourse $X$ is characterised by a membership function $\mu_{F}: x \rightarrow[0,1] . \mu_{F}(x)$ $\in[0,1]$ is the membership value of element $x$ in $F$, from full membership to full nonmembership through all intermediate values. The objects satisfy imprecise properties to varying membership values between the entire unit interval $[0,1]$. The fuzzy set $\mathrm{F}$ is usually denoted by the set of order pairs $F=\left\{\left(x, \mu_{F}(x)\right), x \in X\right]$. For a finite set $X=\left\{x_{1}, \ldots, x_{l}\right\}$, the fuzzy set $F$ of $X$ may also be represented in the form of ' + ' denoting the union of its constituent singletons.

$$
F=\mu_{F}\left(x_{1}\right) / x_{1}+\ldots+\mu_{F}\left(x_{i}\right) / x_{l} \text { or, } F=\sum_{k=1}^{1} \mu_{F}\left(x_{k}\right) / x_{k},
$$

where ' $\mu_{F}\left(x_{k}\right) / x_{k}$ ' is the pair 'membership value/element'. For an infinite set $X$, the fuzzy subset $F$ on $X$ may be represented in the form of an integral denoting the union of the fuzzy singletons $\mu_{F}(x) / x, x \in X$.

$$
F=\int_{x \in X} \mu_{F}(x) / x
$$


Because $\mu_{F}$ maps all real number $x \in X$ into the entire unit interval $[0,1]$, a fuzzy set corresponds to continuouslyvalued logic; An object can be partially belongs to a fuzzy set. The membership value $\mu_{f}(x)$ may be interpreted as the degree of compatibility of $x$ with the concept represented by $F . \mu_{H^{\prime}}(x)$ may also be interpreted as the degree of possibility of $x$ given $F$.

\section{Definition 3 Composition of Fuzzy Relations}

A fuzzy relation was defined as a fuzzy collection of order pairs. If $X=\{x\}$ and $Y=\{y\}$ are collection of objects denoted generically by $x$ and $y$, then a fuzzy binary relation, $W$, from $X$ to $Y$ (or, equivalently a fuzzy binary relation in $X \cup Y$ ) is a fuzzy subset of $X \times Y$ characterised by a membership (characteristic) function $\mu_{W}$ which associates with each pair $(x, y)$ its membership value $\mu_{W}(x, y)$ in $W . \mu_{W}(x, y)$ is the strength of the relationship between $X$ and $Y$. When $X$ and $Y$ are finite sets, $\mu_{W}$ may be represented by a relation matrix whose $(x, y)$ th element is $\mu_{W}(x, y)$. Similarly, if $Y=\{y\}$ and $Z=\{z\}$ are collection of objects denoted generically by $y$ and $z$, then a fuzzy binary relation $R$ from $Y$ to $Z$ characterised by a membership function $\mu_{R}$. $\mu_{R}$ may be represented by a relation matrix whose $(y, z)$ th element is $\mu_{R}(y, z)$.

The composition of $W$ followed by $R$ is denoted as $U=$ WoR, $\mathrm{U}$ where is a fuzzy binary relation from $x$ to $z$ characterised by a membership function $\mu_{\mathrm{U}}$ which associates with each pair $(x, z)$ its membership value $\mu_{\mathrm{U}}(x, z)$ in $U$. o denotes generalized matrix multiplication. Then, $\mu_{U}(x, z)=\underset{y \in Y}{\square}\left(\mu_{w}(x, y){ }^{*} \mu_{k}(y, z)\right), x \in X, z \in Z$, where

$\left(\square,{ }^{*}\right)$ is the pair of operation defining 0. o can be any of the four product operations defined as follows:

$$
\begin{aligned}
& o=(\Sigma, \wedge), \text { sum-min composition, } \\
& o=(\vee, \bullet), \text { max-product composition, } \\
& o=(\vee, \wedge), \text { max-min composition, } \\
& o=(\Sigma, \bullet), \text { sum-product composition. }
\end{aligned}
$$

In practical decision making application, we recommend the sum-product composition, because this composition is more sensitive accepting all the strong membership values as well as weak membership values of elements during composition. We therefore will not miss fragmentary information during composition.

In this paper, the relation matrix for the composition of $W$ and $R$ is given by the sum-product composition of the relation matrices for $W$ and $R$.

$$
\mu_{U}(x, z)=\sum_{y \in Y}\left(\mu_{W}(x, y), \mu_{R}(y, z)\right), x \in X, z \in Z
$$

If $W$ is a annary fuzzy relation (a fuzzy vector) over $y$,

$$
\mu_{U}(z)=\sum_{y \in Y}\left(\mu_{W}(y) \cdot \mu_{R}(y, z)\right), z \in Z
$$

\section{Definition 4. Numerical Transformation of Fuzzy Utilities}

In the case of the countable universe of discourse $X=\left\{x_{k}{ }^{\circ} k=1, \ldots, l\right\}$. The fuzzy utility $\mathrm{U}$ is expressed as the union of its constituent fuzzy singletons $\mu_{\mathrm{U}}\left(x_{k}\right) / x_{k}, k=1, \ldots, l . \mu_{\mathrm{U}}\left(x_{k}\right)$ is the membership value of $x_{k}$ in $\mathrm{U}$. We extend Yager's ranking function [10] in order to transform the fuzzy utility, $\mathrm{U}=\sum_{k=1}^{l} \mu_{\mathrm{u}}\left(x_{k}\right) / x_{k}$, into a numberical score. The ranking function is defined as

$$
S_{\mathrm{u}}(z)=\sum_{k=1}^{l} g\left(x_{k}\right) \cdot \mu_{\mathrm{u}}\left(x_{k}\right) / \sum_{k=1}^{l} \mu_{\mathrm{u}}\left(x_{k}\right)
$$

where $S_{\mathrm{V}}$ is the transformed numerical score of $\mathrm{U}$ and $g\left(x_{k}\right)$ is the weight function measuring the weight of the element $x_{k}$. The score of $\mathrm{U}, S_{\mathrm{U}}$, may be seen as the weighted mean value of $\mathrm{U}$. All the fuzzy utilities of 
alternatives can be transformed into numerical scores, which can be used to compare the strength of satisfaction of alternatives to the decision maker(s).

\subsection{QFD Fuzzy Evaluation and Prioritisation Approach}

In real environment, the relationship ratings can not be evaluated precisely as described in the traditional QFD evaluation matrix in section 2, therefore, the relationship ratings in this new QFD fuzzy evaluation matrix are expressed and derived by using fuzzy set theory. The relationship rating of design attribute $A_{j}$ against customer requirement $C_{i}$ is fuzzy and is expressed by different membership values on different appraisal levels. The appraisal level set range is from $P_{1}$ to $P_{l}$, with $P_{1}$ representing the level of greatest relationship and $P_{l}$ representing the level of smallest relationship. The membership values range from 0 to 1 . The membership value of $A_{j}$ against $C_{l}$ is supported $f_{i j 1}$ on appraisal level $P_{1}$ and $f_{i j k}$ on appraisal level $P_{k}$. Thus, the fuzzy set "Relationship of $C_{i}$ and $A_{j}$ " on support set $P, P=\left\{P_{i}, P_{2}, \ldots, P_{l}\right\}$ may be defined by

$$
\text { Relationship }=f_{i j 1} / P_{1}+f_{i j 2} / P_{2}+\ldots+f_{i j i} / P_{1} \text {. }
$$

For convenient calculation, we let $\sum_{k=1}^{l} \dot{f}_{i j k}=1, i=1, \ldots, m, j=1, \ldots, n$. The characteristics of the design attribute $A_{j}$ with respect to all customer requirements $C=\left\{C_{1}, C_{2}, \ldots, C_{m}\right\}$ on all appraisal leveis $P=\left\{P_{1}, P_{2}, \ldots\right.$, $\left.P_{1}\right\}$ can be characterised by fuzzy sets shown as follows:

$$
\begin{aligned}
& A_{j}=\left\{\left(C_{1},\left[f_{1 j 1} f_{1,2} \ldots f_{1 j j}\right]\right)\right. \text {, } \\
& \left(C_{2},\left[f_{2 j 1} f_{2 j 2} \ldots f_{2 j l}\right]\right), \\
& \text {... } \\
& \begin{array}{lll}
\cdots & \\
\cdots & \text { ats }
\end{array} \\
& \left.\left(C_{m},\left[f_{m j 1} f_{m j 2} \ldots f_{m j l}\right]\right)\right\} \text {, }
\end{aligned}
$$

where $f_{j j k}$ is the membership value of the design attribute $A_{j}$ with respect to the customer requirement $C_{i}$ on the appraisal level $P_{k} ; i=1 \ldots m, j=1 \ldots n$ and $k=1 \ldots l$. By using the matrix notation, the fuzzy rating of $A_{j}$ with respect to all $C_{i}$ in $C$ and on all appraisal levels $P_{k}$ in $P$ can be represented by the following matrix:

$$
R_{j}=\left|\begin{array}{c}
f_{1 j 1} f_{1,2} \ldots f_{1 j l} \\
f_{2 / 1} f_{2 j 2} \ldots f_{2 j t} \\
\ldots \\
\ldots \\
f_{m j 1} f_{m j 2} \cdots f_{m j l}
\end{array}\right| .
$$

Assume that each customer requirement $C_{j}$ in $C$ has a different importance weighting $W_{j}^{\prime}$ in $W$. The values of importance weighting set $W$ derived by the AHP method can be described by $W \div\left[w_{1} w_{2} \ldots w_{m}\right], w_{l} \in[0 ; 1]$ and $\sum_{i=1}^{m} w_{l}=1$. The importance weightings and the fuzzy relationship.ratings are composited and thus the fuzzy utility of $A_{j}$ can be obtained as: 


$$
\begin{aligned}
\mathrm{U}_{j} & =W^{\circ} R_{j} \\
& =\left[\begin{array}{llll}
w_{1} & w_{2} \ldots w_{m}
\end{array}\right]^{\circ}\left|\begin{array}{c}
f_{1 j 1} f_{1 / 2} \ldots f_{1 j l} \\
f_{2,1} f_{2, j 2} \ldots f_{2 j l} \\
\ldots \\
\ldots \\
\ldots \\
f_{m j 1} f_{m j 2} \ldots f_{m j l}
\end{array}\right| \\
& =\left[f_{j 1} f_{j 2} \ldots f_{j l}\right],
\end{aligned}
$$

where $f_{j k}=\sum_{i=1}^{m} w_{i} \cdot f_{i j k}, j=1 \ldots n, k=1 \ldots l$.

Assume that each appraisal level $P_{k}$ has a different appraisal value $p_{k}$. The appraisal values of appraisal level set $P$ can be described by a vector $P=\left[p_{1} p_{2} \ldots p_{l}\right]$. The global appraisal score of $U_{j}$, also called as absolute importance rating of $A_{j}$, can be obtained as:

$$
S_{j}=\sum_{k=1}^{l} p_{k} \cdot f_{j k} / \sum_{k=1}^{l} f_{j k}, j=1, \ldots, n .
$$

The normalised importance rating $S_{j}$ can be derived according to the following formula:

$$
S_{j}^{\prime}=S_{j} / \sum_{j=!}^{n}\left|S_{j}\right|
$$

\subsection{Illustrated Example}

$$
\therefore
$$

s

Now consider the QFD evaluation matrix illustrated in above example and re-evaluate the software design plan by using QFD Fuzzy evaluation matrix (see Figure 4). 'The strength of relationship of attribute $A_{j}$ is judged against seven customer requirements $C_{1}, C_{2}, \ldots, C_{7}$. The fiuzzy. ratings of $A_{j}$ may be described by membership values on five different appraisal levels, $P_{1}, P_{2}, \ldots, P_{s}$. Assume that the characteristics of the design attributes $A_{1}$ with respect to all customer requirements $C_{i}, i=1, \ldots, 7$; on levels $P_{k}, k=1, \ldots, 5$, can be described by a fuzzy set shown as follows:

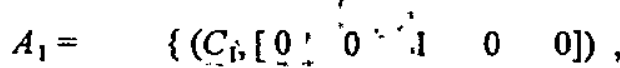

$$
\begin{aligned}
& \left(C_{2},\left[\begin{array}{lllll}
0^{*} & 0 & 0.2 & 0.8 & 0
\end{array}\right)\right. \text {, } \\
& \left(C_{3},\left[\begin{array}{lllll}
0.9 & 0.1 & 0 & 0 & 0
\end{array}\right]\right) \text {, } \\
& \left(C_{4},\left[\begin{array}{lllll}
0 & 0 & 0.4 & 0.6 & 0
\end{array}\right]\right) \text {, } \\
& \left(C_{5},\left[\begin{array}{lllll}
0 & 0 & 1 & 0 & 0
\end{array}\right]\right), \\
& \left(C_{6},\left[\begin{array}{lllll}
0 & 0 & 0.5 & 0.5 & 0
\end{array}\right]\right) \text {, } \\
& \left(C_{7},\left[\begin{array}{lllll}
0 & 0 & 0.5 & 0.5 & 0
\end{array}\right)\right\} \text {. }
\end{aligned}
$$

By using the matrix representation method, $A_{1}$ can be represented by the matrix as follows:

$$
R_{1}=\left|\begin{array}{ccccc}
0 & 0 & 1 & 0 & 0 \\
0 & 0 & 0.2 & 0.8 & 0 \\
0.9 & 0.1 & 0 & 0 & 0 \\
0 & 0 & 0.4 & 0.6 & 0 \\
0 & 0 & 1 & 0 & 0 \\
0 & 0 & 0.5 & 0.5 & 0 \\
0 & 0 & 0.5 & 0.5 & 0
\end{array}\right|
$$

From Eq.(3), the fuzzy utility of $A_{1}$ can be obtained as follows: 

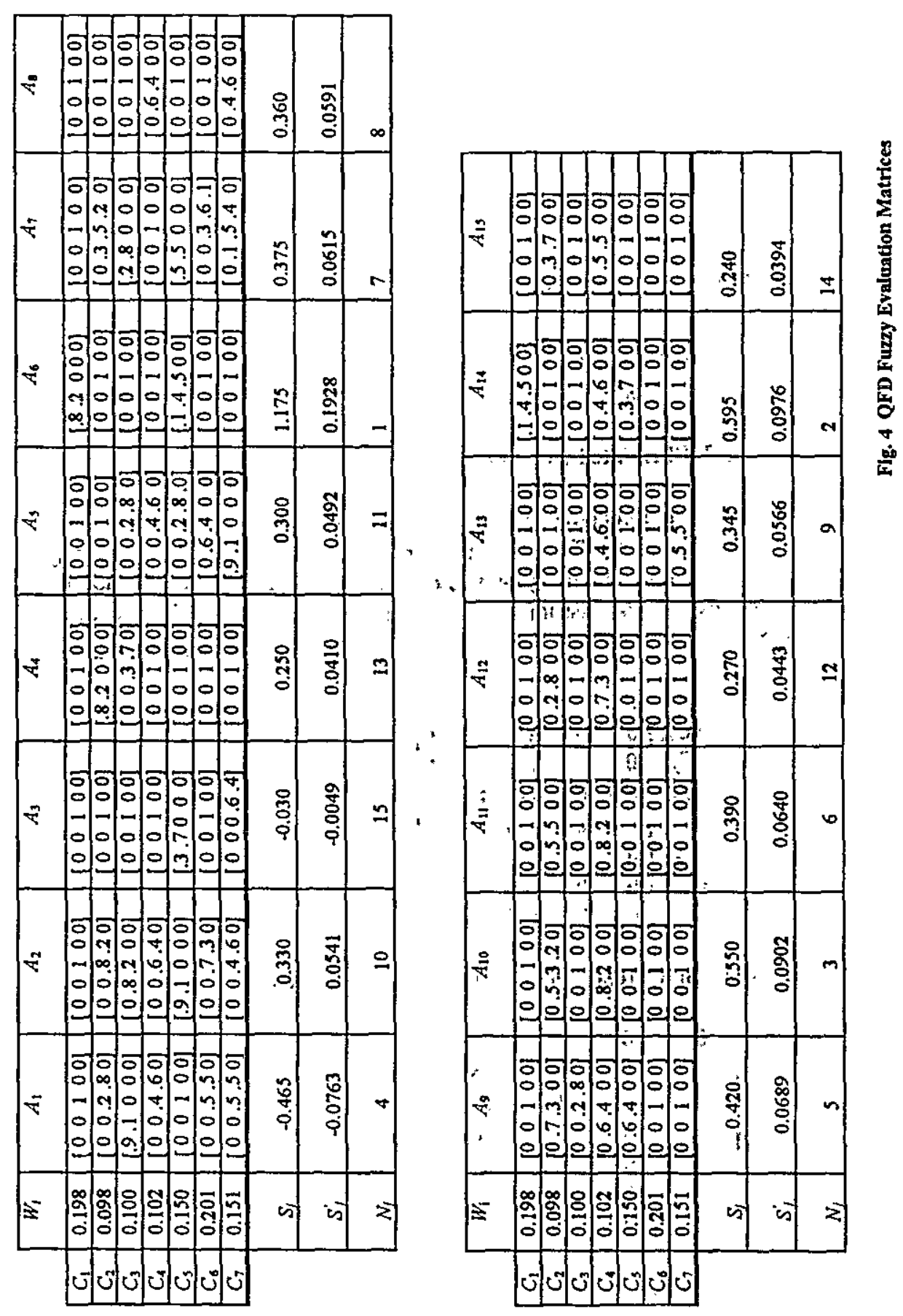

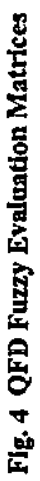




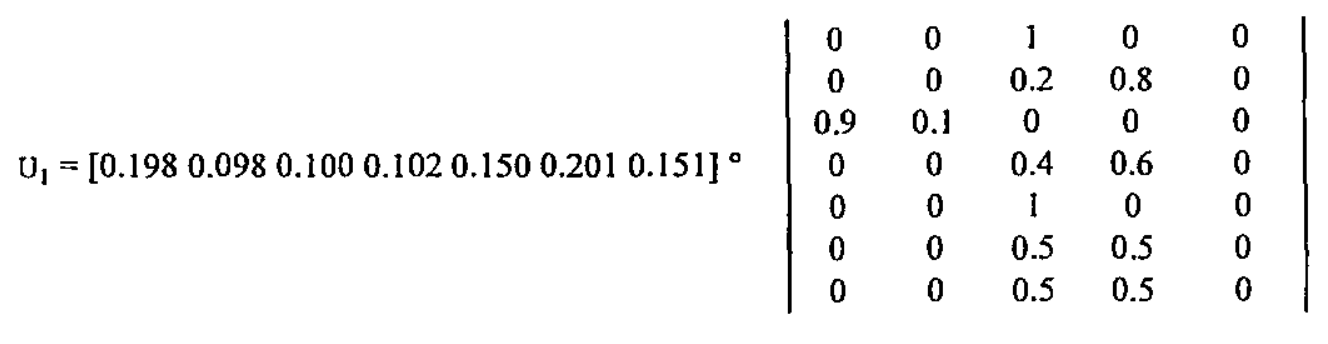

$$
\begin{aligned}
& =[0.09,0.01,0.584,0.316,0]
\end{aligned}
$$

The values of appraisal level $\left\{p_{1}, p_{2}, p_{3}, p_{4}, p_{5}\right\}$ can be quantified through the use of $\{5,3,0,-3,-5\}$ scales to denote "strong positive relationship", "positive relationship", "weak relationship", "negative relationship" or "strong negative relationship" respectively. Therefore, from Eq.(4), the absolute importance rating of $A_{1}$ can be obtained as follows:

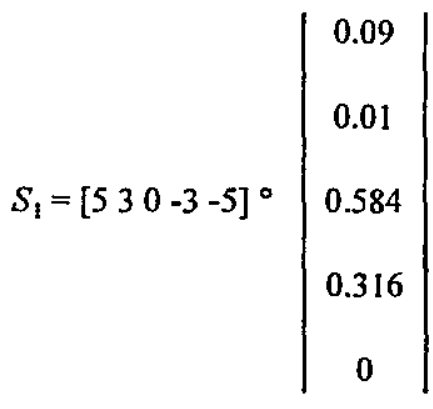

$$
\begin{aligned}
& =-0.465
\end{aligned}
$$

In a similar manner, we can obtain the absolute importance ratings of other attributes as illustrated in Figure 4.

From Eq.(5), the normalised importance rating of $A_{1}$ can be obtained as $S_{1}{ }^{\circ}=-0.465 / 6.095=-0.0763$. In a similar manner, the normalised importance ratings of other attributes can be derived as illustrated in Figure 4.

Therefore, the order of ranking of the design attributes is derived as illustrated in Figure 4. The top six design attributes that most likely to impact a positive or negative change in customer satisfaction are

$\operatorname{Correctness}\left(A_{6}\right)>\operatorname{Verifiability}\left(A_{14}\right)>\operatorname{Expandability}\left(A_{10}\right)>\operatorname{Integrity}\left(A_{1}\right)>\operatorname{Maintainability}\left(A_{9}\right)>\operatorname{Flexibility}\left(A_{11}\right)$.

The top six attributes that constitute $58.98 \%$ of the total normalised importance value (100\%) should determine the areas of focus for customers satisfaction. According to the order of raking, more design attributes can also be incorporated and calculated to increase the total importance value which impact the change in customer satisfaction, either positively or negatively.

\section{Conclusions}

In order to early evaluate and assure the quality of software products, we have illustrated the application of QFD evaluation matrix in software design plan. Through the application of the QFD evaluation matrix, the quality design attributes which present the extra attraction for customer satisfaction are identified. Furthermore, for overcoming the limitation of the crisp ratings, we have proposed a new QFD fuzzy evaluation matrix to prioritise quality design attributes more realistically because the fuzzy ratings in the new evaluation matrix reflect all the membership values on all appraisal levels. Thus, the sharp and unambiguous boundary dividing members from nonmembers of one specific appraisal level can be eliminated. After we prioritise the quality design attributes, critical design attributes with the most positive or negative leverage for satisfying customer requirements can be identified.

\section{References}

1. B.W. Boehm, J.R. Brown, J.R.Kaspar, M. Lipow, G.J. MacCleod, and M.J. Merrit, Characteristics of Software Quality (North-Holland, 1978). 
2. J.A. McCall, P.K. Richards, and G.F. Walters, Factors in software Quality, Vols. I, II, III, (US Rome Air Development Centre Reports NTIS AD/A-049014, 015, 055, 1977).

3. M.S. Deutsch and R.R. Willis, Software Quality Engineering: A Total Technical and Managemen Approach (Prentice-Hall, Englewood Cliffs, New Jersey, 1988).

4. Y. Akao (ed.), Quality Function Deployment-Integrating Customer Requirements into Product Design (Productivity Press, Cambridge, Massachusetts, 1990).

5. J.L. Bossert, Quality Function Deployment: A Practitioner's Approach (ASQC Quality Press, Milwaukee, Wisconsin, 1991).

6. J.R. Hauser and D. Clausing. "The House of Quality," Harvard Business Review (May-June 1988) 63-73.

7. M. Philips, P. Sander and C. Govers, "Policy Formulation by Use of QFD Techniques: A Case Study," Int.

J. Quality and Reliability Management 11(5)(1994) 59-80.

8. L. Saaty, The Analytic Hierarchy Process (McGraw-Hill, New York, 1980).

9. L. A. Zadeh, "Fuzzy sets", Inform. Control 8 (1965) 338-356.

10.G. Bortolan and R. Degani, "A Review of Some Methods for Ranking Fuzzy Subsets," Fuzzy sats and Systems 15(1985) 1-19. 